\title{
Stiffness-matched segmented metallic guidewire for interventional cardiovascular MRI
}

Burcu Basar ${ }^{1,2^{*}}$, Adrienne E Campbell-Washburn', Toby Rogers', Merdim Sonmez ${ }^{1}$, Anthony Z Faranesh', Kanishka Ratnayaka', Robert J Lederman', Ozgur Kocaturk ${ }^{1,2}$

From 18th Annual SCMR Scientific Sessions

Nice, France. 4-7 February 2015

\section{Background}

Conductive guidewires and intravascular catheters are at risk of RF-induced heating under MRI [1]. Heating is found predominantly at the tip of conductive wires [2], and is modulated by wire diameter, length and insulation thickness [3]. Non-conductive materials, such as polymer, impart unsatisfactory mechanical properties on guidewires in terms of flexibility, stiffness, and torquability, for navigating tortuous cardiovascular structures and for safely delivering catheter devices.

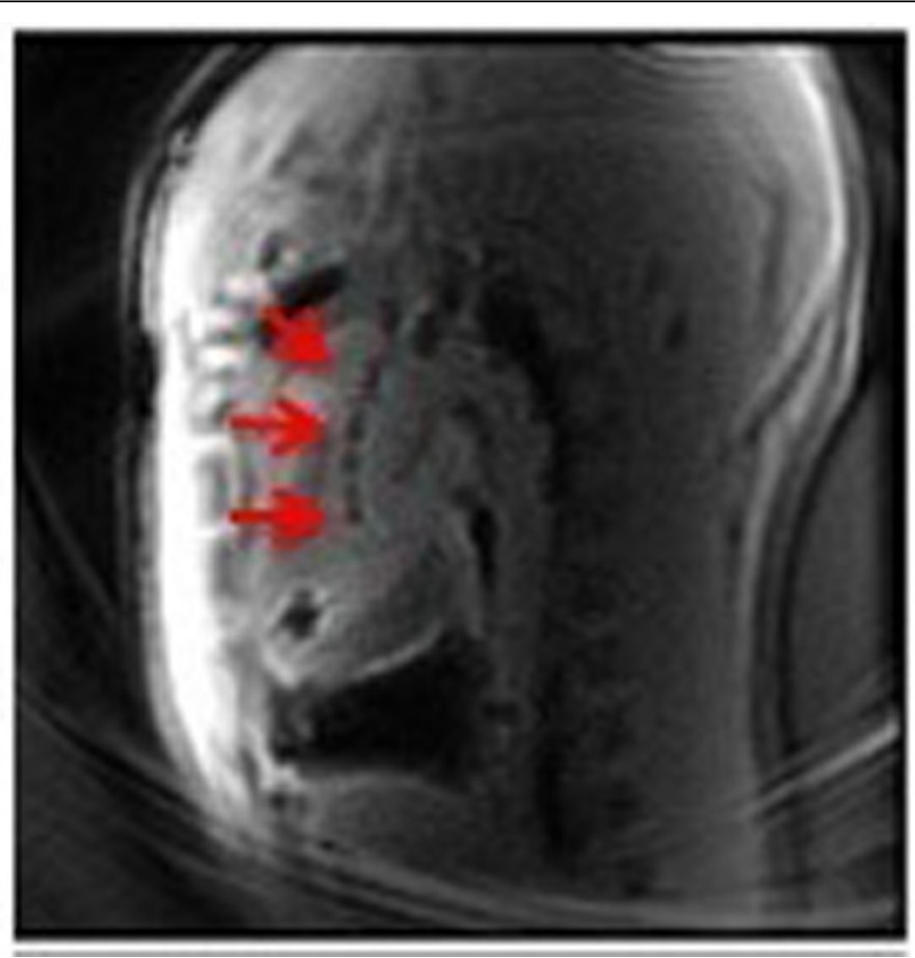

Figure 1 In vivo left heart catheterization in swine using MRI safe guidewire design. Iron-oxide markers on device tip shown with red arrows. TE/TR=1.9/4.2 ms, slice thickness=6mm, matrix=192x192, flip angle=15.

'Division of Intramural Research, National Heart, Lung and Blood Institute,

National Institutes of Health, Bethesda, MD, USA

Full list of author information is available at the end of the article

(c) 2015 Basar et al; licensee BioMed Central Ltd. This is an Open Access article distributed under the terms of the Creative Commons 


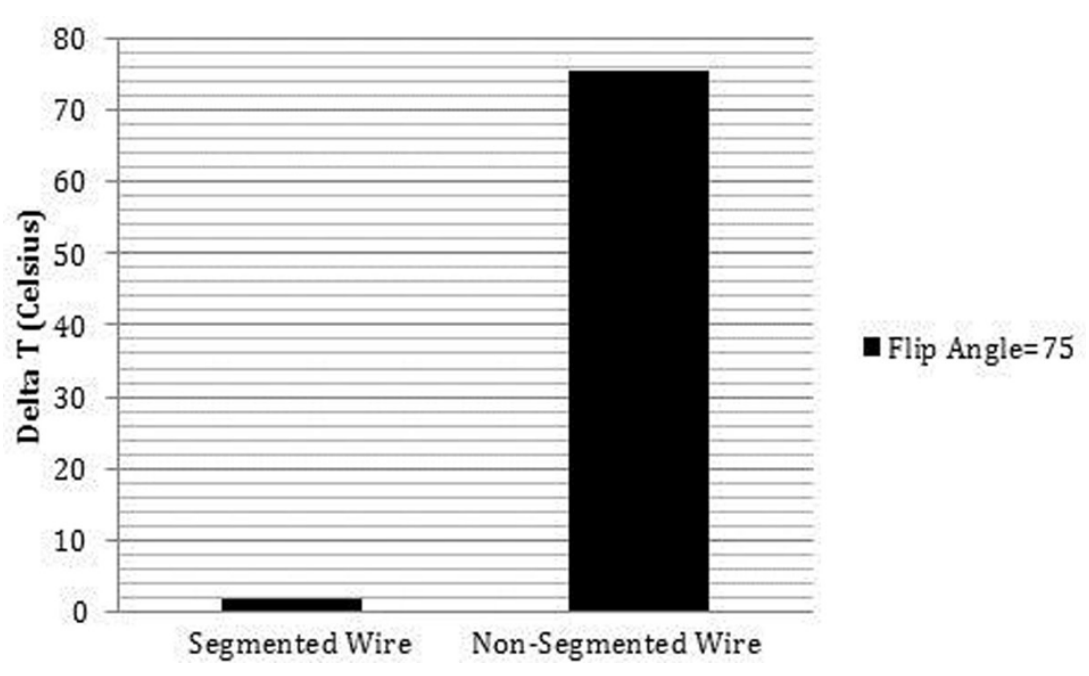

Figure 2 Deviation from baseline temperature readings acquired over 30 seconds prior to scanning during in-vitro heating experiments.

We developed a novel MRI guidewire design that avoids RF heating yet preserves the mechanical features of conventional X-ray guidewires. Short non-resonant segments of nitinol are connected using stiffnessmatched insulated notched couplers, preventing standing wave formation yet appearing mechanically indistinguishable from nitinol guidewires.

\section{Methods}

The core of a passive $120 \mathrm{~cm}$ guidewire was constructed from nitinol rod segments shorter than a quarter-wavelength in vivo at $1.5 \mathrm{~T}(10 \mathrm{~cm})$. The segments were joined by electrically insulated nitinol tubes. A surrounding outer braided polymer enhanced torque response, insulation and safety. The distal tip was insulated using nonbraided polymer and low durometer thermoplastic polymer for flexibility and trackability. Iron-oxide markers created MRI susceptibility artifacts for enhanced visualization in vivo in a swine (Fig 1).

RF heating was measured in an ASTM F2182 gel phantom. Tip and shaft temperature was measured using a fiber optic probe (OpSense) at 1.5T (Aera, Siemens) [4]. Heating was measured at high flip angle $\left(75^{\circ}\right)$ bSSFP (TR/TE, 2.88/1.44 ms; thickness, $6 \mathrm{~mm}$; FOV, $350 \times 350 \mathrm{~mm}$; matrix, $192 \times 144)$ and compared to a custom non-segmented nitinol core wire with identical jacketing serving as a control.

\section{Results}

The segmented MRI guidewire exhibited a maximum temperature increase of $1.6^{\circ} \mathrm{C}$ at the tip, compared with $74^{\circ} \mathrm{C}$ for the non-segmented comparator, during a $60 \mathrm{~s}$ scan at a flip angle of $75^{\circ}$ (Fig 2). Systematic temperature measurements along the shaft detected negligible heating, confirming successful electrical insulation by the inter-segment connectors. Trackability in a tortuous vascular phantom resembled commercial comparators (Glidewire, Terumo).

\section{Conclusions}

We demonstrate a simple and intrinsically safe new design for passive metallic MRI guidewires. The guidewire exhibits negligible heating at high flip angles in conformance with ISO standards $\left(<2^{\circ} \mathrm{C}\right)$ [5], yet mechanically resembles a high-performance conventional metallic guidewire. This may represent a significant advance once applied in clinical MRI catheterization.

\section{Funding}

Supported by NHLBI Z01-HL006041. BB, OK, and RJL are co-inventors on patent applications assigned to $\mathrm{NIH}$.

\section{Authors' details}

${ }^{1}$ Division of Intramural Research, National Heart, Lung and Blood Institute, National Institutes of Health, Bethesda, MD, USA. ${ }^{2}$ Institute of Biomedical Engineering, Bogazici University, Istanbul, Turkey.

\section{Published: 3 February 2015}

\section{References}

1. Yeung: Magn Reson Imaging 2002, 47:187-193.

2. Nitz: J Magn Reson Imaging 2001, 13:105-114.

3. Weiss: Magnet Reson Med 2005, 54:182-189.

4. Sonmez: J Cardiov Mag Reson 2012, 14:38.

5. ISO/TS 109742012.

doi:10.1186/1532-429X-17-S1-P414

Cite this article as: Basar et al:: Stiffness-matched segmented metallic guidewire for interventional cardiovascular MRI. Journal of Cardiovascular Magnetic Resonance 2015 17(Suppl 1):P414. 